\title{
Guidelines and conditions for use of health claims in Denmark
}

\author{
By Heddie Mejborn, Lars Ove Dragsted, Jørn Dyerberg, Bente Koch, \\ Morten Poulsen, Ellen Trolle and Lars Ovesen
}

\begin{abstract}
๑• A working group was established by the Danish Food Administration to propose guidelines and conditions for the use of health claims on foods. This article is an overview of the proposal from the working group.

The scientific evidence behind a health claim must be based on a systematic review of all scientific publications with relevance for the claim in question. Three requirements must always be fulfilled: 1) the health claim must be based on the total scientific evidence; 2) the health claim must be based on experiments on human subjects and be in accordance with the results of these experiments; and 3) consensus with respect to the scientific documentation must be reached among qualified experts.

The designs of human research can be ranked due to their strength of scientific proof. Health claims should always have their basis in high-ranking scientific
\end{abstract}

ABSTRACT

\section{Introduction}

A health claim can be defined as any representation used for marketing, that states, suggests, or implies that a relationship exists between a food, a nutrient, or a dietary component and health and/ or disease, including disease prevention.

In order not to mislead the consumers the claim must be scientifically documented. This paper is based on the report from a working group that was established by the Danish Food Administration to propose guiding rules for the scientific documentation of health claims and conditions for their use.

Use of claims, especially health claims, is considered important for marketing of functional foods. There is no specific regulatory framework for functional foods, rather they are considered as common foods, and thus subject to the same rules regarding claims as common foods are.

\section{Status of regulation of claims in Denmark}

Codex Alimentarius defines a claim as "any representation which states, suggests or implies that a food has particular qualities relating to its origin, nutritional properties, nature, processing, composition or any other quality." A nutrition claim is defined as: "any representation and any advertising message which states, suggests or implies that a foodstuff has particular nutrition properties with respect to its energy and/or nutrient content."

Nutrition claims are allowed within the EU - and in Denmark, provided that the guidelines accepted at the Codex Alimentarius Commission in 1997 are complied with, e.g., that the claims are consistent with national nutrition policy and support that policy. Nutrition claims include:

Heddie Mejborn ${ }^{1}, \mathrm{MSc}, \mathrm{PhD}$, Lars Ove Dragsted ${ }^{1}, \mathrm{MSc}$, PhD, Jørn Dyerberg ${ }^{2}$, Prof, Dr Med, Bente Koch ${ }^{1}$, MSc, Morten Poulsen ${ }^{1}$, MSc, Ellen Trolle ${ }^{1}$, MSc, Lars Ovesen $^{1}, \mathrm{MD}$, Head.

${ }^{1}$ Food Administration of Denmark, Søborg, Denmark

${ }^{2}$ The Danish Council of Nutrition, Søborg, Denmark

Correspondence: Lars Ovesen, Institute of Food Research and Nutrition, 19 Mørkhøj Bygade, DK-2860 Søborg, Denmark, evidence. Often it is also required to know the mecha$\operatorname{nism}(s)$ of the claimed effect, the minimum and maximum intake of the food and the frequency of its use to obtain the effect, and whether the effect is relevant only for a subset of the population. The report gives a number of examples of fictive health claims and their scientific requirements.

The report states several general requirements for the use of health claims, which must be fulfilled, to secure that consumers are not mislead, and principal requirements for contents of fat, sugar and salt in foods that can carry a claim.

Finally, suggestions for the wording of three claims are given, based on the opinions from expert groups.

Key words: Health claims, functional foods, legislation, scientific assessment

\section{Nutrient content claims, which describe the level of a nutrient} in a food.

Examples: "low fat", "sugar free", "high in fibre", "with 100 to 200 gram fresh strawberries you cover your daily need for vitamin C".

2. Comparative claims, which compare the nutrient level and/or energy value of two or more foods.

Examples: "light", "less fat", "reduced fat content", "increased fibre content".

3. Nutrient function claims, which describe the physiological role of a nutrient in growth and development and other normal functions of the body.

Examples: "calcium aids in the development of strong bones and teeth", "iron is a factor in red blood cell formation", "protein helps build and repair body tissue", "folic acid contributes to the normal growth of the fetus".

4. Claims related to dietary guidelines or healthy diets.

Examples: "eat more fish; the Food Administration recommends that you eat 200 to 300 gram fish per week", "milk is a good source of calcium and other nutrients; the Food Administration recommends that you ingest half a litre of lean milk or milk products/day".

Nutrition claims can only be used in relation to energy, protein, carbohydrate, fat and components thereof, plus fibre, and sodium, and vitamins and minerals for which Nutrient Reference Values (NRVs) have been established in the Codex Guidelines for Nutrition Labelling. Claims referring to the natural content of vitamins and minerals are only accepted if the micronutrients account for a significant amount, which is defined as at least $15 \%$ of NRV in $100 \mathrm{~g}$ or $100 \mathrm{ml}$ or per one-serving packet.

Furthermore, Codex proposes the following conditions for using nutrient function claims:

1. Only those nutrients for which an NRV has been established in the Codex Guidelines on Nutrition Labelling or those nutrients, which are mentioned in officially recognised dietary guidelines, should be the subject of a nutrient function claim.

2. The food for which the claim is made should be a significant source of the nutrient in the diet. 
3. The claim should be based on scientific consensus, which is supported by the competent authority.

4. The claim should not imply or include any statement to the effect that the nutrient would afford a cure, or a treatment for or protection from disease.

Nutrient function claims have until now not been allowed in Denmark, because they have been considered as health claims. It has, however, been legal to use claims 1., 2., and 4. in Denmark, as long as the claims were not misleading.

Pursuant to $\$ 20$, article 2 of The Danish Food Act, it is not allowed in advertisements and the like to mention that "ingestion of the product can prevent, relieve or be beneficial towards human disease or disease symptoms". This provision effects the EU Council Directive, article 2, in the Danish legislation, after which "the labelling and methods used must not attribute to any foodstuff the property of preventing, treating or curing human disease, or refer to such properties". Thus, health claims are presently illegal in Denmark even if they are scientifically substantiated.

At the meeting in Codex Alimentarius' labelling committee in 1999 in Ottawa, Canada, two alternative definitions of health claims were suggested: 1) any claim establishing a relation between a food or a constituent of that food and health, [whether it is good health or a condition related to health] or 2) any claim that suggests that a food or a constituent of that food has an impact on health. Health claims are not included in Codex Alimentarius "Guidelines for Use of Nutrition Claims". It should be emphasised that in the relevant Codex committees it is still discussed whether to put a ban on health claims, or to accept their use.

\section{Scientific methods}

When using a health claim it is important to ensure that scientific documentation for the statement is valid. For that purpose several types of experimental methods exist. Below follows a short description of the different methods.

\section{In vitro methods}

In vitro methods are biological test methods that are carried out in a test tube whether performed by use of tissue cultures, cell cultures or cell fragments, or bacterial cultures. In vitro experimental strategies can be divided in three types: 1) screening tests, 2) analytical tests and 3) mechanistic tests. In vitro methods are usually much cheaper than animal and human experiments, and therefore very useful in a testing process. The weakness of the methods is primarily that they cannot be used to evaluate an overall effect in the whole organism, and they can only be used in connection with mechanistic effects that are already known.

\section{Biomarkers}

Biomarkers refer to minimally invasive methods, which establish the presence of a certain substance or a certain biochemical, biological or physiological effect in a living system (1-3). Thus, they indicate the presence of a substance or an effect in a biological system, and the underlying assumption is that the presence or level of the marker is relevant to the state of health. Most biological markers are at the developmental stage, and the number of validated markers is indeed very low. It is expected, however, that biomarker experiments will become increasingly important in the design and implementation of larger animal and human investigations (4).

\section{Animal experiments}

Experiments in laboratory animals are very important for the health evaluation of foods and chemical compounds. They have their strength specifically in safety evaluations, where international guidelines exist for several validated experimental methods (5). Animal experiments can be performed in strictly controlled environments and can have a considerable duration in relation to the normal life span of the animals. This makes animal experiments useful for testing hypotheses about health effects of specific food components, impossible to carry through in humans. Besides, as animal experiments leave the possibility of a more extensive sample material, they can also be used to increase our understanding of physiological, biochemical, and genetic effects of food components. The most obvious weakness of animal experiments is that biological systems may be different between animals and humans, and one must always consider if such differences can affect the interpretation of the results (6).

\section{Human studies}

Studies in humans are necessary to verify that results obtained in vitro or in animals also apply to humans $(7,8)$. Basically, two types of studies are relevant: observational studies and experimental studies (9-11). In observational, also called epidemiological studies, the investigator merely makes observations on individuals without experimental manipulation, while in experimental studies the investigator manipulates the allocation of individuals to different groups, i.e., the researcher has direct control over many aspects of the investigation.

Observational studies are useful to identify associations. Criteria for evaluating the causal significance of an association between a factor and an effect include the consistency, strength, specificity, temporality and coherence of the association (see below). An association between a factor and an outcome, which satisfies these criteria can be taken as a strong, if not absolute, indication of causality. Observational studies with respect to nutrition are particularly difficult to interpret because of the complicity of nutrients and foods in a diet, and the fact that many other factors related or unrelated to nutrition (confounders) may influence health outcome.

Observational studies can be divided in two main types: descriptive epidemiological studies (ecologic, migrant, correlation and time-series studies) and analytical epidemiological studies. The analytical studies include cross-sectional studies, the prospective (cohort follow-up or longitudinal) studies and the retrospective case-control studies.

Experimental trials can be used to test hypotheses generated as a result of one of the other types of investigations. Double-blindness and randomisation are both vital to avoid bias and hence to achieve validity of the trial, but other factors are also relevant, e.g., adequate sample size, clearly defined end points and appropriate statistics. The strength of the blinded and randomised trial - i.e., the controlled trial - is that it can prove whether a certain intervention has an effect. Consequently the controlled trial is considered the gold standard for the verification of causality. However, the applicability of the results from a controlled trial to the (usually much larger) target population requires a representative study population, i.e., a random sample. Use of biomarkers in controlled trials can help demonstrate the mechanism of action of a dietary component on body physiology, biochemistry, or on the genes and the size of the variation within a population, thus contributing to a better safety evaluation.

\section{Weighing of evidence and conditions for documentation}

The scientific evidence must always be based on a systematic review of relevant scientific literature according to scientifically accepted principles (12).

The total evidence requires careful weighing of both the type and the strength of the contributions coming from the kinds of studies 
mentioned above. It takes considerable experience to do this. Therefore, the level of evidence is usually arrived at by several consenting experts. There are, however, some conditions, which, when present, strengthen the evidence for a connection between diet and health (the design of the controlled trial takes several of these conditions into account):

1. Consistency. The findings must be demonstrated more than once, and preferably by other scientists, in different human populations and/or animal species, under different conditions and at different times.

2.Strength and quality. The study design must be suitable for examining the actual connections, and the results must be statistically significant.

3. Biological plausibility. Preferably, the connection should be supported by a mechanistic theory, consistent with the physiological and biochemical circumstances. In vitro methods, animal experiments and biomarkers contribute considerably to that evidence.

4.Dose-response relationship. Preferably, a dose-response relationship should be demonstrated and the minimal effective dose should be determined. Animal experiments are well suited for that, but observational and experimental studies and metaanalyses can contribute.

5. Temporality. A change in diet must be followed by a change in health outcome. The temporality requirements are fulfilled in controlled experiments in animals and humans.

6. Specificity. The evidence is strengthened when the (degree of) change in health outcome is specific for the dietary factor in question. All kinds of experiments can contribute to fulfil this condition.

Despite the fact that the different human study methods have their independent merits and to some extent raise different questions and therefore give different answers - it is possible to rank the individual methods according to their scientific strength for proving diet-health relations. A ranking of the methods after this principle can be referred to as "the hierarchy of evidence" (Table 1). Animal studies can never stand alone in the evaluation of a positive health effect in humans (contrary to the case for negative effects), but in principle are comparable to controlled trials. If the knowledge about the animal model as a model for humans is less than complete, then evidence from animal studies typically ranks lower than studies performed in humans. In vitro methods alone rank very low as scientific evidence, but can support other results to make the total evidence stronger.

Meta-analyses - based on experimental as well as observational studies - are so far not sufficiently standardised for use as the only basis for a health claim, but can be used to support the scientific evidence (13).

The scientific requirements for documentation should vary depending on the wording of the health claim but in general certain minimum conditions must always be fulfilled:

1. The claim must be based on all scientific evidence, not only experiments supporting the claim.

2. The claim must be based on human experiments, and the claim must be in accordance with the results of these experiments.

3. A general consensus among independent and qualified scientists must exist.

\section{Risk assessment}

It is very important to perform an overall risk assessment of a dietary component to obtain a comprehensive view both in relation to safety and nutritional aspects. There is no advantage in raising the intake of a food to obtain a modest health advantage, if at the same
Table 1. The hierarchy of evidence for human study methods ranked in descending order of scientific weight.

1A. Systematic review of (several) controlled studies of good quality where all the results point in the same direction.

1B. Isolated or few controlled studies of good quality with narrow confidence intervals where the results point in the same direction.

2A. Systematic review of (several) prospective cohort studies where the results point in the same direction.

2B. Isolated or few prospective cohort studies of good quality, and isolated randomised controlled studies with broad confidence intervals.

2C. Systematic review of (several) case-control studies where the results point in the same direction.

2D. Isolated or few case-control studies of good quality.

3A. Correlation studies, cross-sectional studies, time-series studies.

3B. Case reports, and cohort studies and case-control studies of low quality.

time there is risk that adverse effects will overshadow the beneficial effects. As a basis for the risk assessment both results from concrete experiments and considerations of possible side effects based on known effects of the dietary component should be taken into consideration.

Studies in animals and in vitro are important tools for the risk assessment, and for this purpose international validated guidelines are developed (e.g., OECD-guidelines (5)). When extrapolating from observations in animals to humans, differences in reactions between animals and humans and between humans are allowed for. A factor of 10 is often used as a safety margin for each of these two steps (14). I addition, corrections must be made for eventual differences in absorption, metabolism, and excretion, and the magnitude of used dose corrected for weight, or even better corrected for species differences in basal metabolism (15).

To this comes the attention that must be paid to suggestive negative effects in human studies. A negative effect of a dietary component must not be "proven" in the same way as a positive effect, but any suspicion of a negative effect must lead to a closer examination or dismissal of the food in question. This part of the evidence for a health claim (i.e., the safety aspect) can be as extensive as the basis for judging the positive effects.

\section{Examples of claims and requirements for documentation}

In this chapter are given examples of different types of claims used on different kinds of foods, from so-called "common foods" to "functional foods". It must be emphasised, that the examples are imaginary, and the requirements for scientific documentation are indicated to give the reader an impression of their general level. Due to limitations of the different scientific methods, which can be used to examine a health effect of a dietary component, it is not possible to give the quantity or the quality of studies needed to support a claim. The number of studies depends among other things on their scientific quality (including an evaluation of experimental design, representability of the used population, the practical workmanship (randomising, blinding), evaluation of the possibility for bias and confounding, risk for type 1 and type 2 errors, statistical processing), which may vary considerably.

The examples take into account the general principle that the "stronger" the claim, the higher is the scientific evidence. This means, for example, that nutritional claims do not require the same level of evidence as health claims. For the so-called physiological claims (health claims based on the relation between a food or food component and a health related physiological effect) the level of evidence typically must be the same as that required for claims, which mention a reduced risk of a disease. It must be pointed out that it is the opinion of the Danish authorities that physiological 
claims are unwanted, as they are suitable for misleading the consumers. The fact is, it is easy to obtain documentation for an effect on physiological parameters without showing the importance for effect on disease prevalence. The consumer, however, will easily interpret the claim as if eating the actual food will reduce the risk for disease.

The following documentation is mandatory:

1. The food bearing the claim can contribute positively to good health, when eaten as a part of a normal diet.

2. The claimed effect can be achieved by intake of a reasonable amount of the food in question.

3. The effect is maintained through a prolonged period by the continuous intake of the food in question.

Furthermore, knowledge about the mechanism behind the claimed effect may be needed, the minimum and maximum, and frequency of intake to obtain the effect, and whether special population groups alone or particularly will benefit from the food. In vitro studies and animal studies are not included in all examples below, but their applicability, and their strengths and weaknesses in relation to the documentation of a health claim have already been mentioned.

- Product: Juice processed under high pressure (a product where desired components are preserved).

Claim: Contains a high natural content of flavonoids, which reduce the risk for heart disease.

Evaluation: Powerful health claim with mentioning of disease, which will require documentation according to level $1 \mathrm{~A}$, supported by mechanistic tests. The demonstrated mechanisms must not imply a risk, shown in guideline tests in experimental animals.

- Product: Tomato with increased content of lycopene (a product, which has been changed by genetic modification).

Claim: The high content of lycopene helps strengthen the antioxidative defence.

Evaluation: A so-called functional claim, which requires documentation according to level $1 \mathrm{~B}$. At the same time the increased content of lycopene could be viewed as an addition, requiring special documentation with respect to safety.

- Product: Iodine-fortified salt (a classical fortified product).

Claim: Fortified with iodine, which prevents goitre.

Evaluation: Health claim mentioning a disease. Iodine is an essential nutrient for which an NRV is established, and its role in preventing goitre has been known for decades. The claim must be documented to be nutritionally relevant, which will require documentation according to $1 \mathrm{C}$. Furthermore, post-marked survey according to level $2 \mathrm{~B}$ is compulsory.

- Product: Yoghurt with bifido bacteria (a product modified by means of microorganisms).

Claim: Promotes gut health and improves the immune system.

Evaluation: Two claims where "healthy gut" can be considered a functional claim and the effect on "the immune system" a health claim. The evaluation takes origin in the "stronger" claim on the effect on the immune system, which requires documentation corresponding to $1 \mathrm{~B}$. In addition, a microbiological safety evaluation of the bifido-culture is required.

- Product: Bread containing fish oil/n-3 fatty acids (a product with new ingredients added).

Claim: Reduces the triglyceride in the blood.

Evaluation: Health claim that requires documentation according to level $1 \mathrm{~B}$. The safety evaluation in connection with the increased intake of fish oil will be included.

- Product: Tomato with an added gene for angiotensin-I-converting enzyme inhibitor-production (a completely new product produced by means of genetic modification).

Claim: Contains active ingredients, which decrease blood pressure. Evaluation: Should be considered a health claim, as the claim is expected to appeal to persons with the disease: increased blood pressure. Documentation according to level $1 \mathrm{~A}$ will be required.

- Product: Seaweed with a natural content of special fibres (a completely new food).

Claim: Contains soluble fibres that decrease the blood cholesterol level.

Evaluation: A health claim that will require documentation according to level $1 \mathrm{~B}$. With respect to safety the product will be assessed according to the criteria given for novel foods, e.g., a 'substantial equivalence' evaluation, where the product is compared to known products.

- Product: Fruits and vegetables (common foods).

Claim: A high intake of fruits and vegetables reduces the risk of several cancers.

Evaluation: A generic health claim. Documentation can not be produced by means of randomised controlled trials. Will require documentation according to level $2 \mathrm{~A}$.

\section{Practical handling of scientific documentation}

The Danish Food Administration has set up a steering committee, which has been given the responsibility to make a list of proven and acceptable health claims, and to propose conditions for their use, and to establish disqualifying criteria. Further, it is the task of the steering group to appoint expert groups, to evaluate the strength of the scientific documentation behind a diet/health relation.

By way of introduction 3 expert groups were appointed to evaluate the following diet/health relations:

1.Intake of fruits and vegetables and risk of heart disease and cancer. 2.Intake of fish and risk of heart disease.

3.Intake of calcium and vitamin D and risk of osteoporosis.

The mandate for the scientific working groups was:

"The scientific working group (expert group) shall examine the connection between a food group, a food, a food component, or a nutrient and health/disease, and conclude if there is sufficient scientific documentation for the connection to be true at a realistic level of intake. In addition, the expert group, if possible, should give an upper intake level for the food or food component. The expert group must indicate if certain population groups differ in relation to need or tolerance. Finally, the expert group shall propose a wording for the claim".

The expert groups were appointed in April, 2000 and finalised their work in June, 2000. The expert groups did not have access to the requirements for scientific documentation given in this report. The main conclusions from the expert groups were the following:

Fruits and vegetables and risk of heart disease and cancer "...it is convincingly demonstrated that a diet rich in fruits and vegetables reduces the risk of heart disease and cancer. No single fruit or vegetable, or even groups of fruits or vegetables, can be assigned special positive effects. Neither can specific food components be considered beneficial. However, there is a considerable risk of confounding from a healthy life style. The expert group, therefore, finds that there is no basis for disjoined claims on fruits and vegetables, hence claims must always include recommendations for an overall healthy life style."

\section{Fish and risk of heart disease}

"Epidemiological studies suggest that an increased fish intake (up to 40-60 g of unspecified fish daily, similar to about $1 \mathrm{~g}$ of marine n-3 fatty acids) is associated with reduced cardiac death ... in highrisk groups. The association, however, can not be shown in low-risk groups. Despite lack of intervention studies in healthy population 
groups the general population is recommended a daily intake of 40 $60 \mathrm{~g}$ of fish of mixed type (around $300 \mathrm{~g}$ weekly). Even if fish, particularly fat fish, can contain pollutants, a daily intake of 40-60 $\mathrm{g}$ is not associated with any health risk. Intervention studies with fish/fish oil capsules indicate that intake of 4-7 g marine n-3 fatty acids per week improves the prognosis in patients with previous coronary thrombosis. A daily intake of $1 \mathrm{~g}$ n-3 fatty acids ( $7 \mathrm{~g}$ per week) is recommended for patients with coronary heart disease. This appears to reduce the risk of cardiac death."

\section{Calcium and vitamin $D$ and risk of osteoporosis}

This expert group has not expressed a final conclusion, and the minute was accompanied by a note of dissent. The group treated the two nutrients separately, even if it is recognised that their connection to osteoporosis is closely related. For calcium it is stated that low intake in childhood and adolescence is assumed to impede the development of bones, and that "circumstances that result in calcium deficiency is associated with increased risk of osteoporosis, as well as it has been shown that calcium supplementation to the elderly decreases fracture risk". And finally that "a vitamin D supplement of $15-20 \mu \mathrm{g}(600-800 \mathrm{iu})$ per day in combination with calcium has been shown to decrease the bone fracture risk in elderly, which has not been clearly shown for vitamin D alone."

\section{General conditions for use of health claims}

It is essential that the use of health claims is based on the principle that it is the total diet and not a single food that is important for health. Claims, therefore, must be given within the context of the total diet. It is particularly important that claims on individual products are not misleading.

For health claims to be allowed, certain requirements must be laid down, partly concerning the nature and the composition of the claimed food, partly concerning the scientific documentation of the claim. The requirements mentioned below are divided into general requirements (which always must be fulfilled), and principal requirements for the composition of the foods (exemptions can be given).

\section{General requirements, which must be fulfilled}

1)The claim must be true and not suited for misleading.

2)The claim must be based on all scientific evidence, and be in accordance with the Danish authorities' requirements for documentation.

3)The claim must be in accordance with the Danish nutrition and health policies.

4) The claim must be stated within the context of the total diet, and the food carrying the claim must contribute significantly to a healthy diet.

5) The claim must be true for a realistic intake of the food. If possible, information of the level of intake to achieve the claimed effect should be given as well as an upper limit of intake if available.

6)If the claimed effect is caused by a specific food substance, the food which is carrying the claim must contain an amount of the substance that lives up to the claim, and the level of the substance must be labelled.

7) Claims must not be used for foods with properties that increase the risk of disease.

8)Labelling must contain information of target group. The general requirement is that the food can be eaten by all, and if there are population groups that should not eat the food, it must be considered if the food in question may carry a health claim.

9) The claim must not imply that the food can prevent ("prevent" means "totally prevent", while expressions giving the impression of risk reduction (help to prevent) are allowed) or treat or cure a disease.
Principal requirements for foods carrying a bealth claim Fat content:The principal requirements for contents of fat, sugar and salt in foods that can carry a claim are:

Fat: $\quad$ Meat and meat products: maximum $10 \mathrm{~g} / 100 \mathrm{~g}$.

Poultry: maximum $10 \mathrm{~g} / 100 \mathrm{~g}$.

Fish: natural content.

Bread: maximum $5 \mathrm{~g} / 100 \mathrm{~g}$.

Flour, grains and oats: natural content.

Fruits and vegetables: natural content.

Other products: maximum $3 \mathrm{~g} / 100 \mathrm{~g}$ or $1.5 \mathrm{~g} / 100 \mathrm{~mL}$.

Sugar: Products must not contain added sugar, including sugar alcohols, oligosaccharides and artificial sweeteners.

Salt: Natural foods (non-manufactured): natural content.

Bread: $1.5 \mathrm{~g} / 100 \mathrm{~g}$.

Other products: $2.5 \mathrm{~g} / 100 \mathrm{~g}$.

Nutritional quality: To guarantee that only nutritious foods can carry a health claim, requirements about minimum content without fortification, corresponding to $10 \%$ of NRV of one or more essential nutrients, must be met.

\section{Suggestions for claims wording}

Based on the opinions from the expert groups the steering committee suggests the following claims:

- A high intake of fruits and vegetables contributes to a healthy diet and may reduce the risk of heart disease ana many cancers.

The claim can be used on all kind of fruits and vegetables without added fat or sugar.

- Fish are rich in several important nutrients (including omega-3 fatty acids) and contribute to a healthy diet. One or two fish meals per week may reduce the risk of heart attack.

The claim can be used on all kinds of fish and certain fish products.

- The content of calcium and vitamin D in the diet is important for formation and maintenance of healthy bones. This food [label may be mentioned] contributes significantly to the calcium intakel vitamin $D$ intake.

The claim can be used on low fat milk products, certain vegetables, fish, and certain kinds of meat.

\section{REFERENCES}

1. Griffith J, Duncan RC, Hulka BS: Biochemical and biological markers: implications for epidemiologic studies. Arch Environ Health 1989;44:375-81.

2. Henderson RF, Bechtold WE, Bond JA, Sun, JD: The use of biological markers in toxicology. Crit Rev Toxicol 1989;20:65-82.

3. Hermus RJJ, van't Veer P, Kok FJ: Biomarkers: signals between health and disease. In: Somogyi JC and Hötzel D eds. Early recognition and intervention in nutrition-dependent diseases, Bibl. Nutr. Dieta, Karger, Basel, 1992;1-16.

4. WHO regional office for Europe: Guiding principle for the use of biological markers in the assessment of human exposure to environmental factors: an integrative approach of epidemiology and toxicology. Toxicology 1995;101:1-10.

5. OECD guidelines for testing of chemicals, section 4 - Health effects. Environment Directorate, Organisation for Economic Co-operation and Development, Paris, 1996.

6. Calabrese EJ: Principles of animal extrapolation. John Wiley \& Sons, New York, 1983.

7. Clydesdale FM: What scientific data are necessary? Nutr Rev 1996;54:S195-S198.

8. Clydesdale FM: A proposal for the establishment of scientific criteria for health claims for functional foods. Nutr Rev 1997;55:413-22.

9. Freudenheim JL: A review of study designs and methods of dietary assessment in nutritionel epidemiology of chronic disease. J Nutr 1993;123:401-5.

10. ILSI monograph: Nutritional epidemiology, possibilities and limitations. ILSI Europe, 1996.

11. Tarasuk VS, Brooker A-S. Interpreting epidemiologic studies of diet-disease relationships. J Nutr 1997;127:1847-52.

12. Meade MO, Richardson WS: Selecting and appraising studies for a systematic review. Ann Intern Med 1997;127:531-7.

13. Jüni P, Witschi A, Block R, Egger M: The hazards of scoring the quality of clinical trials for meta-analysis. JAMA 1999;282:1054-60.

14. Renwick AG: Data-derived safety factors for the evaluation of food additives and environmental contaminants. Food Additives Contam 1993;10:275-305.

15. Travis CC, White RK, Wards, RC: Interspecies extrapolation of pharmacokinetics. J Theor Biol 1990;142:285-304. 